\title{
An Interactive Visualization of the Catalogue of Galactic Planetary Nebulae
}

\author{
J. Köppen ${ }^{1} \&$ A. Acker ${ }^{1}$ \\ ${ }^{1}$ Observatoire Astronomique de Strasbourg, Strasbourg, France
}

\begin{abstract}
At http://astro.u-strasbg.fr/ /koppen/png/png.html you'll find the first release of an interactive version of the StrasbourgESO Catalogue of Galactic Planetary Nebulae by Acker et al. (1992). The observational data of 1185 planetary nebulae can be inspected in detail numerically. Any two properties can be plotted against each other, with interactive zooming, graphical marking of groups of nebulae with certain properties, and identification and display of the data of any individually selected object. Maps of the sky - as well as of the space of any two parameters - can be displayed for any third parameter. Nebulae may be selected according to their names or observed parameters. This interactive catalogue is an application of the JAVA applet CatPlot which also permits the user to read in one's own catalogue file and also display images and $\mathrm{x}-\mathrm{y}$ plots (a detailed description may be found in the explanatory pages at http://astro.u-strasbg.fr/ /koppen/catplot /CatPlotHelp.html).
\end{abstract}

